\title{
Multiplex detection of collisional energy transfer using KCSFI $\dagger$
}

\author{
Heiko Frerichs, ${ }^{a}$ Thomas Lenzer, ${ }^{* a b}$ Klaus Luther ${ }^{a}$ and Dirk Schwarzer ${ }^{b}$ \\ ${ }^{a}$ Institut für Physikalische Chemie, Universität Göttingen, Tammannstr. 6, D-37077 Göttingen, \\ Germany. Fax: +49 55139 3150; Tel: +495513912598 \\ ${ }^{\boldsymbol{b}}$ Max-Planck-Institut für biophysikalische Chemie, Abteilung 010, Am Fassberg 11, \\ D-37077 Göttingen, Germany. E-mail: tlenzer@gwdg.de; Fax: +49 551201 1501; \\ Tel: +495512011344
}

Received 15th November 2004, Accepted 24th December 2004

First published as an Advance Article on the web 13th January 2005

\begin{abstract}
A new detection method for obtaining collisional transition probabilities $P\left(E^{\prime}, E\right)$ of highly vibrationally excited molecules in the gas phase is presented. The technique employs energy-selective probing of the time-dependent vibrational population distribution by "kinetically controlled selective fluorescence (KCSF)". We present experimental results for a test system, the collisional deactivation of toluene by argon, where we use the wellknown "kinetically controlled selective ionization (KCSI)" scheme as a reference for comparison. A newly designed setup is employed that allows simultaneous detection of fluorescence and ionization signals under identical experimental conditions ("kinetically controlled selective fluorescence and ionization = KCSFI"). For the system toluene + argon it is demonstrated that KCSF and KCSI yield identical results. A rate-equation model is presented to understand common features and differences of both approaches. The fluorescence detection scheme shows promise for future investigations on collisional energy transfer. The experimental setup is simpler, because it requires no additional ionization wavelength. This will hopefully give access to the $P\left(E^{\prime}, E\right)$ of systems where, e.g., ionization schemes are difficult to implement due to short wavelengths required for the ionization step. A few examples will be outlined briefly.
\end{abstract}

\section{Introduction}

Direct experimental techniques to study collisional energy transfer (CET) of highly vibrationally excited molecules are of central importance to characterize the energy dependent efficiency of collisions and understand the mechanisms of CET. ${ }^{1,2}$ Early methods like "infrared fluorescence (IRF)",3,4 or "ultraviolet absorption (UVA") ${ }^{5,6}$ have provided quantities averaged over the whole population distribution during deactivation, mainly the first moment of energy transfer $\langle\Delta E\rangle$ and its energy dependence. With the advent of the method of "kinetically controlled selective ionization (KCSI)" it has finally become possible to measure complete distributions of the central quantity of collisional energy transfer, the collisional transition probability $P\left(E^{\prime}, E\right)$, over wide ranges of vibrational energy and also to improve the accuracy of energy dependent CET parameters like $\langle\Delta E\rangle$ by up to one order of magnitude. ${ }^{7-9} P\left(E^{\prime}, E\right)$ characterizes the energy transfer behavior in a system completely, and is needed as input, e.g., in master equation simulations for combustion models. In this paper we present an alternative approach for detection named "kinetically controlled selective fluorescence (KCSF)". This technique not only provides an equivalent information content on CET, but also opens up new perspectives to investigate systems in which the two-photon ionization technique of KCSI experiences technical problems.

A basic scheme of the KCSF and KCSI pump-probe experiments for toluene is shown in Fig. 1. Briefly, at $t=0$ a quasi-microcanonical population of highly vibrationally excited molecules is prepared in the ground electronic state $\left(\mathrm{S}_{0}{ }^{*}\right)$ by a pump laser (wavelength $\lambda_{0}$ ). After preparation, the $S_{0}{ }^{*}$ molecules are deactivated in a collision cascade with a low

$\dagger$ Dedicated to Prof. Dr Michael Buback on the occasion of his 60 th birthday. pressure (mbar range) bath gas $\mathrm{M}$ and finally reach thermal equilibrium. This is shown on the left side of Fig. 1 by a sequence of schematic population distributions. They relax and broaden in $\mathrm{S}_{0}$ during the sequence of collisions with $\mathrm{M}$, before they finally return back into the ambient temperature Boltzmann distribution.

In the KCSI process the $\mathrm{S}_{0}{ }^{*}$ molecules are detected by a pulsed laser resonant two-photon ionization process after a time delay $\Delta t$. This is shown on the right side of the figure. By absorption of a photon with the wavelength $\lambda_{1}$ the molecules in $\mathrm{S}_{0} *$ are pumped into an intermediate electronic state (e.g., $\left.\mathrm{S}_{1}\right)$. A kinetic competition between ionization with a second photon of wavelength $\lambda_{1}$ [rate coefficient $k_{2}(E)$ ] and depletion of the intermediate state population due to nonradiative processes and fluorescence with a total rate coefficient $k_{\mathrm{tot}}(E)=k_{\mathrm{nr}}(E)+$ $k_{\mathrm{F}}(E)$ takes place. The states reached by depopulation of $\mathrm{S}_{1}$ cannot be further ionized at $\lambda_{1}$. The ionization is therefore kinetically controlled by the ratio of $k_{2}(E)$ and $k_{\text {tot }}(E)$. The strong increase of $k_{\text {tot }}(E)$ with $\mathrm{S}_{1}$ excess energy allows the selection of a narrow energy range for the resonant two-step ionization in the $S_{1}$ state (upper grey stripe in Fig. 1). This energy band is projected down onto the $S_{0}$ manifold by the photon energy at $\lambda_{1}$ : As a result an energetic "observation window" $W_{\mathrm{KCSI}}\left(E, \lambda_{1}\right)$ is formed, as indicated by the lower grey stripe in Fig. 1. The lower energy edge of this window is defined by the constraint of the minimum vibrational energy necessary to reach the $S_{1}$ origin with a $\lambda_{1}$ photon. The observation window is shifted by tuning $\lambda_{1}$. Therefore the relaxing population distribution can be monitored at different positions on the $\mathrm{S}_{0}$ energy scale.

For KCSF the population evolution in the relevant energy range (window) of the $S_{0}$ state during time dependent probing of the relaxation is no longer oberved via an additional ionization step. Instead, the fluorescence from the intermediate state $S_{1}$ is detected (Fig. 1). The yield of these fluorescence 


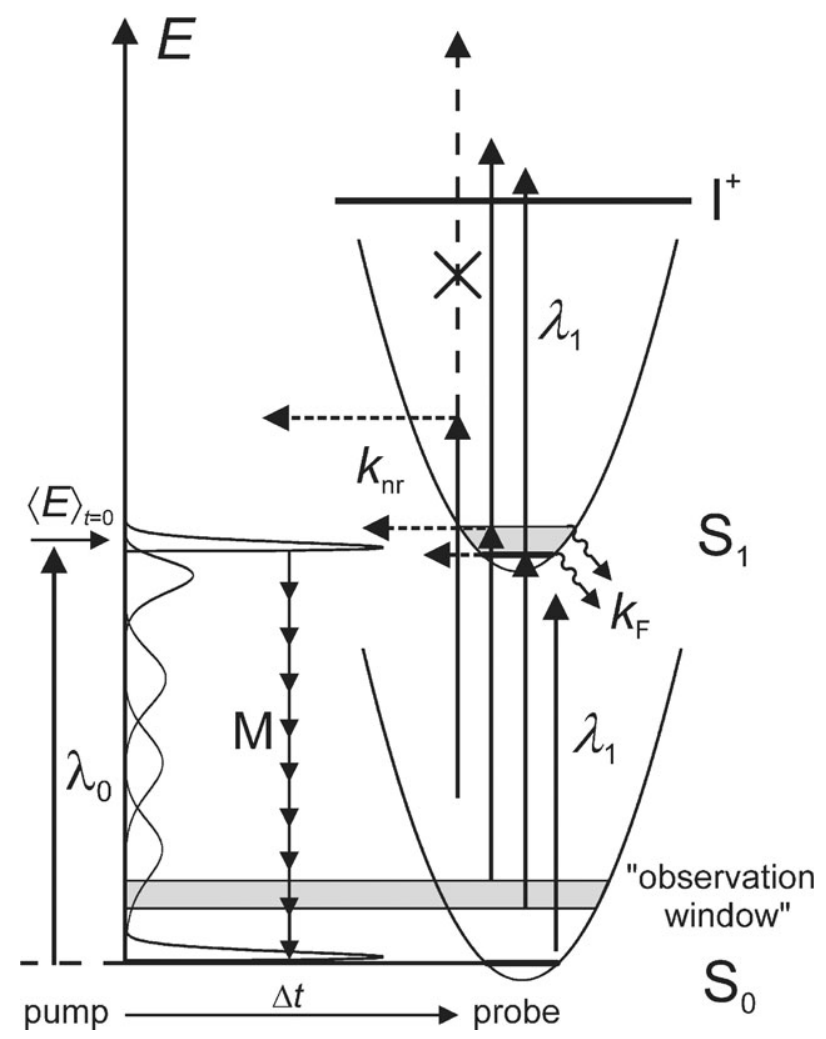

Fig. 1 Simplified KCSFI scheme for toluene. The relaxing distribution of highly vibrationally excited $\mathrm{S}_{0}$ * molecules is monitored through a narrow "observation window" by collecting either fluorescence photons from the $\mathrm{S}_{1}$ state (KCSF) or by detecting ions from a resonant two-photon process via the $\mathrm{S}_{1}$ state (KCSI).

photons is also kinetically controlled, and determined by the energy dependent ratio of the fluorescence rate constant $k_{\mathrm{F}}(E)$ and the total depopulation rate constant $k_{\mathrm{tot}}(E)$, i.e., the energy dependent fluorescence quantum yield $\Phi_{\mathrm{F}}(E)$ in the $\mathrm{S}_{1}$ state. This gives rise to a $\mathrm{KCSF}$ "observation window" $W_{\mathrm{KCSF}}\left(E, \lambda_{1}\right)$, which not necessarily has the same shape as the KCSI window function $W_{\mathrm{KCSI}}\left(E, \lambda_{1}\right)$. A more detailed discussion of the KCSF and KCSI windows on basis of a rate-equation approach will be presented in Section 3.2. The time-dependent KCSF and KCSI signals $I_{\mathrm{F}}(t)$ and $I_{\mathrm{D}}(t)$ are described as a convolution of the respective window function and the time-dependent vibrational distribution $g(E, t)$ in the $\mathrm{S}_{0}$ state:

$$
\begin{aligned}
& I_{\mathrm{F}}(t) \propto \int_{0}^{\infty} g(E, t) W_{\mathrm{KCSF}}\left(E, \lambda_{1}\right) \mathrm{d} E \\
& I_{\mathrm{D}}(t) \propto \int_{0}^{\infty} g(E, t) W_{\mathrm{KCSI}}\left(E, \lambda_{1}\right) \mathrm{d} E
\end{aligned}
$$

Time-resolved fluorescence yields from an electronically excited state in a pump-probe experiment have been reported earlier on, in order to deduce from it vibrational temperatures of ground state trans-stilbene in liquids. ${ }^{10,11}$ Subsequently, Nikowa et al. started attempts to implement the fluorescence analogue of KCSI for the collisional deactivation of transstilbene in liquid $n$-pentane and methanol, and KCSI type signals could be observed. ${ }^{12}$ However, even then the interpretation of these experiments was not straightforward. Obviously, the signals obtained in the condensed phase not only probe the deactivation in the ground electronic state but are also influenced by collisional deactivation in the electronically excited state, due to the very high collision rate under these conditions which can efficiently compete with the $\mathrm{S}_{1}$ fluorescence rate. Moreover a $352 \mathrm{~nm}$ interference filter (bandwidth $22 \mathrm{~nm}$ ) was used, so that a slice of the integral fluorescence was detected. The resulting influence on the shape of the window function $W_{\mathrm{KCSF}}\left(E, \lambda_{1}\right)$ remained still unclear. Consequently, the extraction of well-defined energy transfer properties in the ground electronic state was not possible. The different situation in the condensed phase is also confirmed by very recent measurements for trans-stilbene under gas phase conditions, where deactivation in the $S_{1}$ state can be safely ruled out. Experiments using KCSI and KCSF detection ${ }^{13-15}$ show shapes of relaxation signals and dependences on the probe wavelength that differ from the results in the liquid phase.

Obviously, a direct experimental comparison of the two methods is necessary to evaluate the performance and differences of the KCSF detection scheme relative to the wellestablished KCSI process under identical conditions. For this reason we have constructed a new cell allowing the simultaneous detection of KCSF and KCSI signals (KCSFI). We report here our first results with this setup obtained for the collisional deactivation of highly vibrationally excited toluene ("toluene*") in an argon bath. Interestingly, we find that at our conditions KCSF and KCSI time-dependent signals show practically the same shape. We will interpret both signals in terms of a master equation approach, in which the contributions of different photophysical parameters to the shape of the KCSF and KCSI observation windows will be analyzed by a rate equation model of the competitive processes.

\section{Experimental technique}

The KCSI experiment has been described in detail in our earlier publications, ${ }^{8,9}$ so we focus here only on some specifics of the fluorescence setup. Briefly, the detection flow cell has four ports, two of them carrying window mounts at Brewster's angles each equipped with Suprasil I windows allowing passage of the laser beams into the cell. One port at $90^{\circ}$ with respect to the laser beams features an additional Suprasil I window, through which the fluorescence is monitored by an integrating UV-sensitive photomultiplier (Hamamatsu 1P28A). Efficient reduction of stray light is achieved by Wood's horns (minimizing back reflections from the windows inside the cell) and by two sets of laser baffles. This arrangement allows collection of integral fluorescence signals without the need of using filters for surpressing the laser background signal. This has the additional advantage that one minimizes possible distortions of the KCSF signal, which is emitted in the same wavelength region. Only a single UG11 filter (Schott) was used to prevent visible stray light from entering the detector. This bandpass filter is very well suited for detecting the emission from hot and cold toluene molecules, which occurs in the wavelength range between $260 \mathrm{~nm}$ and $380 \mathrm{~nm} .^{16,17}$ In addition to the fluorescence setup, a sensitive capacitor arrangement was installed inside the cell as described in our earlier KCSI publications, ${ }^{8}$ with a lower detection limit of a few hundred ions.

Highly vibrationally excited toluene molecules ("toluene*") were prepared via excitation of the isomeric precursor 1,3,5cycloheptatriene at $\lambda_{0}=266 \mathrm{~nm}$ using a frequency-quadrupled Nd:YAG laser (Continuum Surelite-I). Detection wavelengths $\lambda_{1}=266-275 \mathrm{~nm}$ were generated by a frequency-doubled excimer pumped dye laser (Lambda Physik LPX 105i and LPD 3002). KCSF and KCSI curves were simultaneously detected in a counterpropagating beam-in-beam arrangement by scanning the delay time between the pump and probe pulses. The ion and fluorescence signals were extracted using series of carefully controlled difference measurements in a fashion very similar to that described previously. ${ }^{8}$

\section{Results and discussion}

\subsection{Comparison and modeling of KCSF and KCSI curves}

Fig. 2 shows the results for the collisional deactivation of highly vibrationally excited toluene with an initial average energy of $49380 \mathrm{~cm}^{-1}$ in 25.5 mbar argon at three different probe wavelengths $\lambda_{1}$. The time axis has been normalized to a "number of collisions" scale using the Lennard-Jones collision 


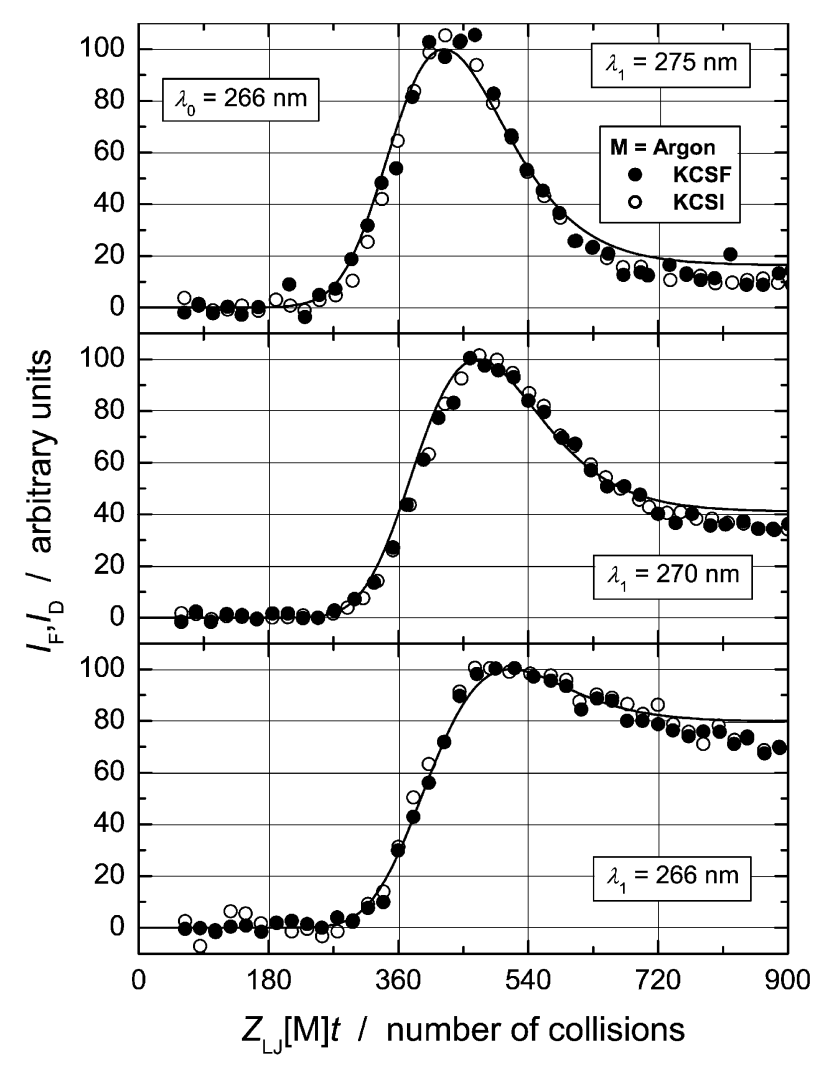

Fig. 2 Comparison of KCSF ( $\bigcirc)$ and KCSI $(\bigcirc)$ signals for the collisional deactivation of toluene* by $\operatorname{argon}\left(P_{\mathrm{tot}}=25.5 \mathrm{mbar}\right)$ for three different observation windows $\left(\lambda_{1}=275,270\right.$ and $\left.266 \mathrm{~nm}\right)$. Excitation of the isomeric precursor CHT at $\lambda_{0}=266 \mathrm{~nm}$. Solid lines: Master equation simulations using a monoexponential $P\left(E^{\prime}, E\right)$ with parametric exponent $Y$ in the argument [eqn. (3)]. The best fit parameters from ref. 9 were employed $\left(C_{0}=43.5 \mathrm{~cm}^{-1}, C_{1}=4.20 \times 10^{-3}\right.$, $Y=0.7)$. Note the identical position and shape of the KCSF and KCSI signals.

number $1.088 \times 10^{7} \mathrm{mbar}^{-1} \mathrm{~s}^{-1} .8$ Interestingly the KCSF and KCSI signals are practically indistinguishable within the accuracy of the experiment, and this is also observed in additional experiments at much lower pressures $(2.8 \mathrm{mbar})$. This result is not necessarily expected a priori (see below). The KCSF signals show the same typical dependence on $\lambda_{1}$ as known from KCSI curves: The fluorescence observation window at the longest wavelength $(275 \mathrm{~nm})$ is highest up on the energy scale, so the KCSF signal appears earliest. The residual fluorescence is due to the overlap of the KCSF observation window with the thermal toluene Boltzmann distribution. With decreasing wavelength, the windows are shifted down the energy scale so the initial rise of the corresponding KCSF signals appears at increasingly later times. In addition, the level of the fluorescence signal after complete deactivation at the end of our measurements increases, because these windows have an increasingly larger overlap with the vibrational Boltzmann distribution of relaxed toluene at the room temperature of the experiment.

Also included in Fig. 2 are master equation simulations (solid lines) using our well-proven empirical minimum parameter expression of the transition probability $P\left(E^{\prime}, E\right)$ for downward collisions (upward collisions defined by detailed balance): ${ }^{7,9}$

$$
\begin{aligned}
P\left(E^{\prime}, E\right) & =\frac{1}{c(E)} \exp \left[-\left(\frac{E-E^{\prime}}{\alpha(E)}\right)^{Y}\right] \\
& =\frac{1}{c(E)} \exp \left[-\left(\frac{E-E^{\prime}}{C_{0}+C_{1} E}\right)^{Y}\right],\left(E^{\prime} \leq E\right)
\end{aligned}
$$

$P\left(E^{\prime}, E\right)$ is monoexponential with an energy dependent parameter $\alpha(E)=C_{0}+C_{1} E$ and a parametric exponent $Y$ in the argument. $Y$ determines the shape of the decay. $\alpha(E)$ describes the width of the CET probability and is therefore directly related to the efficiency of the collisions. $c(E)$ is a normalization constant. In all cases of relaxations from very high levels of vibrational excitation in various bath gases studied so far, the experimental KCSI signals could consistently be modeled with this functional form. For toluene*, the exponents $Y$ vary smoothly with the "size" of the collider, with values between $Y=0.65$ (toluene* + helium) and 1.5 (toluene* $+n$-heptane). The linear dependence of the width parameter $\alpha$ on energy leads to an approximately linear energy dependence of the first moment of energy transfer $\langle\Delta E\rangle$, the average energy transferred per collision, and the square root of the second moment of energy transfer $\left\langle\Delta E^{2}\right\rangle^{1 / 2}$, where the moments $\left\langle\Delta E^{n}\right\rangle$ are defined as:

$$
\left\langle\Delta E(E)^{n}\right\rangle=\int_{0}^{\infty}\left(E^{\prime}-E\right)^{n} P\left(E^{\prime}, E\right) \mathrm{d} E^{\prime}
$$

As one can see in Fig. 2 both the KCSF and KCSI data are well described by our optimized parameters from earlier experiments $\left(C_{0}=43.5 \mathrm{~cm}^{-1}, C_{1}=4.20 \times 10^{-3}, Y=0.7\right) .{ }^{9} \mathrm{KCSI}$ window functions as already presented in our earlier publication were used for modeling. A more detailed discussion of the KCSF and KCSI observation windows can be found in the following sections.

\subsection{Rate-equation model for KCSI and KCSF processes}

To understand the relation between a KCSI signal (ion yield produced by a two-photon ionization via a resonant intermediate state) and a KCSF curve (fluorescence from the resonant intermediate state after one-photon absorption) it is important to compare expressions for the yield of KCSI ions and KCSF fluorescence photons. Because no coherent population-transfer, like e.g., Rabi cycling, occurs under our experimental conditions (weak field limit) a rate-equation approach is adequate. Such descriptions have been frequently applied in the literature to describe inter alia multiphoton and fluorescence processes involving multiple levels. ${ }^{18-22}$ Here, we outline and extend an approach by Bischel and co-workers. ${ }^{18}$ A fourlevel model can be used to describe the toluene system (see also Fig. 1), and the following rate-equations can be set up. Note that in eqn. (5) a misprint in Bischel's original paper is corrected by removing a factor of 2 :

$$
\begin{gathered}
\frac{\mathrm{d} S_{1}}{\mathrm{~d} t}=\frac{I(t)}{h \nu}\left[\sigma_{10} S_{0}-\left(\sigma_{01}+\sigma_{21}\right) S_{1}\right]-k_{\mathrm{tot}} S_{1} \\
\frac{\mathrm{d} I^{+}}{\mathrm{d} t}=\frac{I(t)}{h \nu} \sigma_{21} S_{1} \\
\frac{\mathrm{d} F}{\mathrm{~d} t}=k_{\mathrm{F}} S_{1}
\end{gathered}
$$

$S_{0}, S_{1}$ and $I^{+}$are the toluene populations in a specific vibrational level of the ground electronic state, the first electronically excited state and the ion state, respectively. Toluene molecules can disappear from the vibrational level of the $S_{1}$ state by nonradiative transitions with the rate constant $k_{\mathrm{nr}}$. They are also removed by radiative processes with the rate constant $k_{\mathrm{r}}$. For our case of $\mathrm{S}_{1}$ toluene, only fluorescence is relevant, so that $k_{\mathrm{r}}=k_{\mathrm{F}}$. Consequently, $F$ in eqn. (7) represents the number of photons emitted by toluene molecules which disappear from the vibrational level of the $S_{1}$ state by fluorescence with the rate constant $k_{\mathrm{F}}$. The total depopulation rate constant for the level in $\mathrm{S}_{1}$ is then $k_{\text {tot }}=k_{\mathrm{F}}+k_{\mathrm{nr}}$. All molecules following the radiative and nonradiative channels are lost for the ionization process. $\sigma_{10}$ and $\sigma_{21}$ are the absorption cross sections from the ground electronic state $\left(\mathrm{S}_{1} \leftarrow \mathrm{S}_{0}\right)$ and the first 
electronically excited state $\left(\mathrm{I}^{+} \leftarrow \mathrm{S}_{1}\right.$ ), respectively. $\sigma_{01}$ is the cross section for stimulated emission from the $S_{1}$ down to the $\mathrm{S}_{0}$ state. $I(t)$ and $\nu$ represent the laser intensity and frequency, respectively.

For small laser intensities two approximations can be made which are fulfilled under our conditions. Firstly, depopulation of the $\mathrm{S}_{1}$ level due to stimulated emission and ionization is negligible, so the second term in the squared brackets in eqn. (5) can be removed. Secondly, the $\mathrm{S}_{0}$ state is, in practical terms, not depleted $\left[S_{0}(t) \approx S_{0}(t=0)=\right.$ constant $] .{ }^{18}$ Then eqn. (5) transforms into:

$$
\frac{\mathrm{d} S_{1}}{\mathrm{~d} t}+k_{\mathrm{tot}} S_{1}=\frac{I(t)}{h \nu} \sigma_{10} S_{0}
$$

After introducing the normalized laser intensity $\chi(t)=I(t)$ $A / E$ ( $E=$ laser pulse energy, $A=$ cross section of the laser beam) one obtains:

$$
S_{1}=\frac{E \sigma_{10} S_{0}}{A h \nu} \exp \left(-k_{\text {tot }} t\right) \int_{-\infty}^{t} \chi\left(t^{\prime}\right) \exp \left(k_{\text {tot }} t^{\prime}\right) \mathrm{d} t^{\prime}
$$

For the KCSI process we need to know the ion yield $I^{+}$. Insertion into eqn. (6) and integration yields:

$$
\begin{aligned}
I^{+}= & \left(\frac{E}{A h \nu}\right)^{2} S_{0} \sigma_{10} \sigma_{21} \int_{-\infty}^{+\infty} \chi(t) \exp \left(-k_{\mathrm{tot}} t\right) \\
& \times\left(\int_{-\infty}^{t} \chi\left(t^{\prime}\right) \exp \left(k_{\mathrm{tot}} t^{\prime}\right) \mathrm{d} t^{\prime}\right) \mathrm{d} t
\end{aligned}
$$

One can define the "normalized fractional ionization (NFI)" as: ${ }^{18}$

$$
\mathrm{NFI}=\int_{-\infty}^{+\infty} \chi(t) \exp \left(k_{\mathrm{tot}} t\right)\left(\int_{-\infty}^{t} \chi\left(t^{\prime}\right) \exp \left(k_{\mathrm{tot}} t^{\prime}\right) \mathrm{d} t^{\prime}\right) \mathrm{d} t
$$

In a KCSI experiment one typically uses a laser with a pulse length $t_{\mathrm{FWHM}}$ of about $10 \mathrm{~ns}$. If we assume a Gaussian shape for the laser intensity it is possible to calculate the NFI numerically as a function of the product $k_{\text {tot }} \tau$ (for a Gaussian pulse $\tau=0.753 t_{\text {FWHM }}$ ). The result is shown in Fig. 3 in a double-logarithmic fashion (solid line). Two limiting cases can be distinguished for the NFI. For $k_{\text {tot }} \tau \ll 1$ eqn. (11) simplifies to:

$$
\mathrm{NFI}=\int_{-\infty}^{+\infty} \chi(t)\left(\int_{-\infty}^{t} \chi\left(t^{\prime}\right) \mathrm{d} t^{\prime}\right) \mathrm{d} t=0.5
$$

i.e., the NFI reaches a constant value of 0.5 for arbitrary normalized laser pulses. For the other limit $\left(k_{\mathrm{tot}} \tau \gg 1\right)$

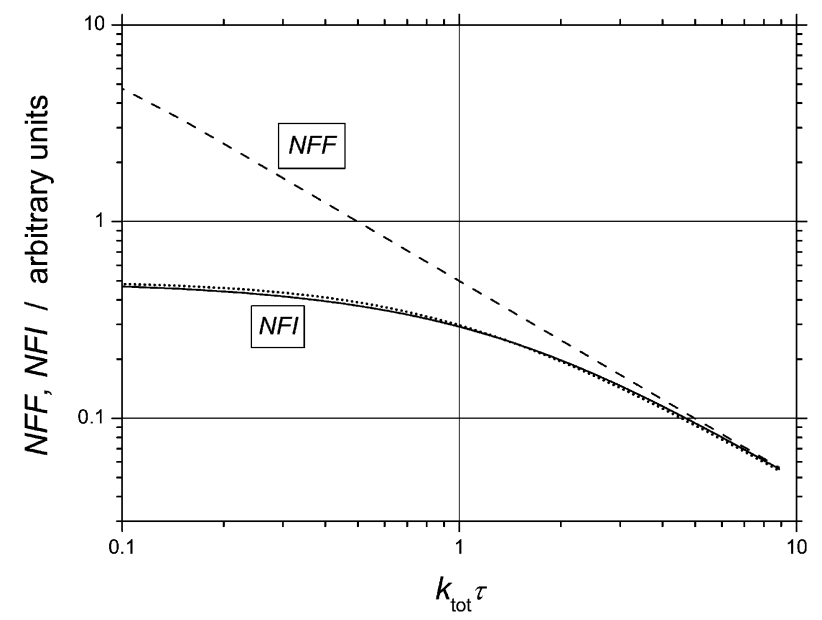

Fig. 3 Comparison of the normalized fractional fluorescence (NFF) and normalized fractional ionization (NFI) for KCSF and KCSI experiments, respectively. Solid line: NFI calculated via eqn. (11) for a Gaussian laser pulse with $t_{\mathrm{FWHM}}=10 \mathrm{~ns}(\tau=7.53 \mathrm{~ns})$. Dotted line: Approximation of the NFI as given in eqn. (14). Dashed line: NFF from eqn. (20), rescaled by a constant factor to demonstrate the same aymptotic behavior of NFI and NFF for large values of $k_{\mathrm{tot}} \tau$. one finds:

$$
\mathrm{NFI}=\frac{1}{k_{\mathrm{tot}}} \int_{-\infty}^{+\infty} \chi^{2}(t) \mathrm{d} t
$$

In this case the NFI is proportional to the inverse of the total depopulation rate constant $k_{\text {tot }}$ in the intermediate state $\mathrm{S}_{1}$. Both limiting cases are clearly visible in Fig. 3. An analytical expression for calculating the KCSI observation windows has proved itself to be very useful in approximating the NFI. It works very well, even for the non-Gaussian pulse shapes of, e.g., typical ns Nd:YAG lasers: ${ }^{18}$

$$
\mathrm{NFI} \approx 0.5\left[1+\left(k_{\mathrm{tot}} \tau\right)^{4 / 3}\right]^{-3 / 4}
$$

This curve is also included in Fig. 3 as a dotted line. Combining eqns. (10) and (14) yields:

$$
I^{+} \propto \sigma_{10} \sigma_{21}\left[1+\left(k_{\mathrm{tot}} \tau\right)^{4 / 3}\right]^{-3 / 4}
$$

Summing up over the full quasicontinuum of vibrational levels, and changing to integration over $E$, one obtains the wellknown KCSI window function $W_{\mathrm{KCSI}}$, as used in the interpretation of our earlier experiments, given here for the case of the one-color two-photon ionization of toluene: ${ }^{7-9,23}$

$$
\begin{gathered}
W_{\mathrm{KCSI}}\left(E\left(\mathrm{~S}_{0}^{*}\right), \lambda_{1}\right) \propto \sigma_{10}\left(\lambda_{1}, E\left(\mathrm{~S}_{0} *\right)\right) \sigma_{21}\left(\lambda_{1}, E\left(\mathrm{~S}_{1} *\right)\right) \\
\times\left[1+\left(k_{\mathrm{tot}}\left(E\left(\mathrm{~S}_{1} *\right)\right) \tau\right)^{4 / 3}\right]^{-3 / 4} \text { for } E \geq E_{\mathrm{low}} \\
W_{\mathrm{KCSI}}\left(E\left(\mathrm{~S}_{0}^{*}\right), \lambda_{1}\right)=0 \text { for } E<E_{\mathrm{low}}
\end{gathered}
$$

$E_{\text {low }}$ is the minimum energy in $\mathrm{S}_{0}$ at which two-photon ionization becomes possible, i.e., $E_{\text {low }}=E_{0-0}\left(\mathrm{~S}_{1}\right)-h c / \lambda_{1}$, where $E_{0-0}$ $\left(\mathrm{S}_{1}\right)$ is the $0-0$ transition energy of toluene $\left(37485 \mathrm{~cm}^{-1}\right){ }^{24}$

In the following we turn to the KCSF process. The number of fluorescence photons $F$ emitted from an intermediate state level was already given in eqn. (7). Combination with eqn. (9) and subsequent integration yields:

$$
F=\frac{E S_{0}}{A h \nu} \sigma_{10} k_{\mathrm{F}} \int_{-\infty}^{+\infty} \exp \left(-k_{\mathrm{tot}} t\right)\left(\int_{-\infty}^{t} \chi\left(t^{\prime}\right) \exp \left(k_{\mathrm{tot}} t^{\prime}\right) \mathrm{d} t^{\prime}\right) \mathrm{d} t
$$

In analogy to the KCSI process one can define a "normalized fractional fluorescence (NFF)" as:

$$
\mathrm{NFF}=\int_{-\infty}^{+\infty} \exp \left(-k_{\mathrm{tot}} t\right)\left(\int_{-\infty}^{t} \chi\left(t^{\prime}\right) \exp \left(k_{\mathrm{tot}} t^{\prime}\right) \mathrm{d} t^{\prime}\right) \mathrm{d} t
$$

For arbitrary values of $k_{\text {tot }} \tau$ the NFF is given by:

$$
\mathrm{NFF}=\frac{1}{k_{\mathrm{tot}}}
$$

i.e., it is proportional to the inverse of the total depopulation rate constant $k_{\text {tot }}$. For comparison we have included the NFF curve in Fig. 3 as a dashed line. If $k_{\text {tot }} \tau$ is large, both the NFI and NFF show the same dependence $\left(\propto k_{\text {tot }}^{-1}\right)$. However, at low $k_{\text {tot }} \tau$, the NFI levels off. This is due to the fact, that in the ionization case the $S_{1}$ population is only probed within the "gate time" (laser pulse width) of the ionization step, whereas fluorescence photons are emitted as long as there is a $\mathrm{S}_{1}$ population. In the limiting case of very large $k_{\text {tot }} \tau$, the population decay in $S_{1}$ happens on a time scale much shorter than the laser pulse length, so practically no fluorescence is emitted after the laser is off, leading to the same $k_{\text {tot }}^{-1}$ dependence of the NFI and NFF.

Substituting eqn. (20) into eqn. (18) yields:

$$
F=\frac{E S_{0}}{A h \nu} \sigma_{10} \frac{k_{\mathrm{F}}}{k_{\mathrm{tot}}}=\frac{E S_{0}}{A h \nu} \sigma_{10} \Phi_{\mathrm{F}} \propto \sigma_{10} \Phi_{\mathrm{F}}
$$

i.e., the number of fluorescence photons is proportional to the absorption cross section $\sigma_{10}$ in $\mathrm{S}_{0}$ and the fluorescence quantum yield $\Phi_{\mathrm{F}}=k_{\mathrm{F}} / k_{\text {tot }}$ in the $\mathrm{S}_{1}$ state. Note that this equation is the KCSF counterpart of eqn. (15). The corresponding window 
function $W_{\mathrm{KCSF}}$ is immediately obtained as:

$$
\begin{gathered}
W_{\mathrm{KCSF}}\left(E\left(\mathrm{~S}_{0}^{*}\right), \lambda_{1}\right) \propto \sigma_{10}\left(\lambda_{1}, E\left(\mathrm{~S}_{0}^{*}\right)\right) \Phi_{\mathrm{F}}\left(E\left(\mathrm{~S}_{1} *\right)\right) \text { for } \\
E \geq E_{\text {low }} \\
W_{\mathrm{KCSF}}\left(E\left(\mathrm{~S}_{0} *\right), \lambda_{1}\right)=0 \text { for } E<E_{\mathrm{low}}
\end{gathered}
$$

Finally we compare the relative yields of the KCSI and KCSF processes. We combine eqns. (10) and (18) to obtain the ratio of the number of ions and fluorescence photons. If both signals are detected under identical conditions (laser energies and beam diameters) one obtains:

$$
\frac{I^{+}}{F} \propto \frac{\sigma_{21}}{k_{\mathrm{F}}} \frac{\mathrm{NFI}}{\mathrm{NFF}}
$$

For the special case of large $k_{\text {tot }} \tau$ it has already been mentioned that the NFI and NFF show the same $k_{\text {tot }}^{-1}$ dependence. eqn. (24) then reads:

$$
\frac{I^{+}}{F} \propto \frac{\sigma_{21}}{k_{\mathrm{F}}}
$$

If the absorption cross section $\sigma_{21}$ for the second step in the KCSI process and the fluorescence rate constant $k_{\mathrm{F}}$ of the $\mathrm{KCSF}$ process are both energy independent or show the same type of energy dependence then the KCSI and KCSF window functions will have an identical shape. In these cases, KCSI and KCSF signals should look exactly the same.

\subsection{Application to toluene}

The assumption of a constant $\sigma_{21}$ probably holds sufficiently well for toluene and other aromatics in the wavelength range of practical interest, as discussed earlier. ${ }^{7,9,25}$ The question, to which regime of $k_{\text {tot }} \tau$ in Fig. 3 the NFI and NFF belong in the case of toluene, is answered by inspection of the energy dependence of $k_{\text {tot }}$. In Fig. 4 a plot of $k_{\text {tot }}$ is shown as a function of $S_{1}$ excess energy. The points represent time-resolved measurements from four different sources. ${ }^{24,26-28}$ The solid line is our earlier fit to these data, ${ }^{9}$ at the time where the measurements from Farmanara et al. ${ }^{28}$ were not yet available. These authors conclude, that $70 \%$ of the toluene molecules with about $12000 \mathrm{~cm}^{-1}$ excess energy ( $S_{2}$ excitation) take a direct internal conversion (IC) pathway $\mathrm{S}_{0} \leftarrow \mathrm{S}_{2}$ with a rate constant $k_{02}=1.4 \times 10^{13} \mathrm{~s}^{-1}, 30 \%$ of the molecules relax via a stepwise IC mechanism $\mathrm{S}_{0} \leftarrow \mathrm{S}_{1} \leftarrow \mathrm{S}_{2}$ with $k_{01}=2.3 \times 10^{11} \mathrm{~s}^{-1}$ for the second step. These newer experimental values are more

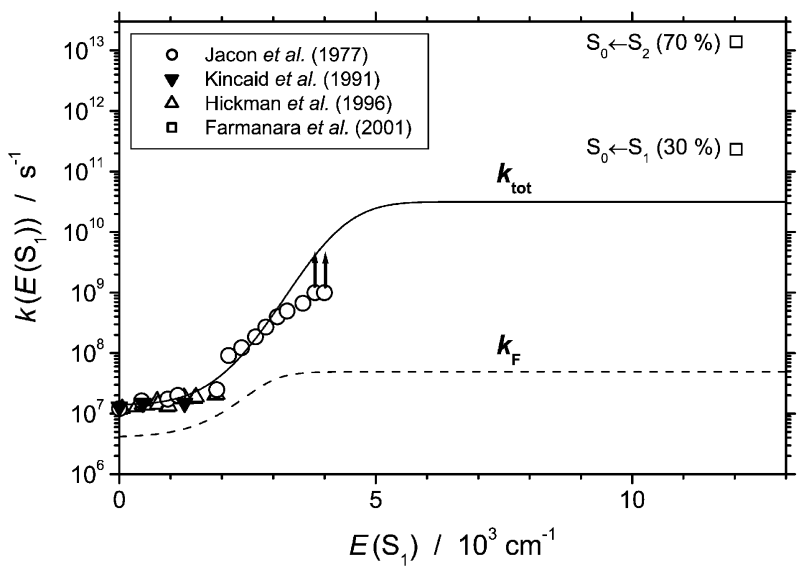

Fig. 4 Energy dependence of the rate constant $k(E)$ for toluene in the $\mathrm{S}_{1}$ state. Time-resolved measurements of $k_{\text {tot }}(E)$ from refs. $24,26,27$ and 28. Solid line: fit from ref. 9, dashed line: estimated fluorescence rate constant $k_{\mathrm{F}}(E)$ which reproduces the experimental fluorescence quantum yields $\Phi_{\mathrm{F}}(E)$ shown in Fig. $5 .^{16,24}$ The experiments of Jacon et al. ( $\bigcirc$ ) have a time resolution of about $1 \mathrm{~ns}$, so their two points at the highest energies are probably lower limits to the true value, as indicated by the two arrows. ${ }^{24}$ than one order of magnitude higher than in our more conservative earlier extrapolation based on the behavior of the related benzene molecule. ${ }^{9}$ It is a nice side effect, that the new data definitely prove that the KCSI (and KCSF) window functions will not "open up" again at high energies, due to the influence of the increase of $\sigma_{10}$ with energy. This was already ruled out indirectly in our earlier paper on the basis of the observed KCSI signal shape. For the following discussion we therefore keep our original function for $k_{\mathrm{tot}}(E)$, which is sufficiently accurate for energies up to $10000 \mathrm{~cm}^{-1}$. Future fits should consider the new data at $12000 \mathrm{~cm}^{-1}$ and will hopefully be based on additional experiments filling up the gap at intermediate $S_{1}$ excess energies. Taking the values from our fit, $k_{\text {tot }}(E)$ varies between $1.4 \times 10^{7}$ and $3.2 \times 10^{10} \mathrm{~s}^{-1}$. For $\tau=$ 7.53 ns (Gaussian laser pulse with $10 \mathrm{~ns}$ FWHM) this corresponds to $k_{\text {tot }} \tau$ values between 0.11 and 238. This means that at low $\mathrm{S}_{1}$ excess energies the $k_{\text {tot }}$ dependence of the NFI and NFF will be different (Fig. 3).

We will now consider the influence of energy dependence of the fluorescence rate constant $k_{\mathrm{F}}$. This is done by using fluorescence quantum yield measurements by Noyes and coworkers, ${ }^{16,24}$ plotted in Fig. 5 as open circles. For comparison, we first calculate a hypothetical $\Phi_{\mathrm{F}}$ curve for an energy independent $k_{\mathrm{F}}=4.1 \times 10^{6} \mathrm{~s}^{-1}$ (solid line). This value is extracted from our fit in Fig. $4\left(k_{\text {tot }}=1.4 \times 10^{7} \mathrm{~s}^{-1}\right.$ at the $\mathrm{S}_{1}$ origin) and an estimate for the fluorescence quantum yield $\Phi_{\mathrm{F}}=$ 0.29 , from ref. 16 mentioned above. As one can see, an energy independent $k_{\mathrm{F}}$ is not consistent with the experimental data in Fig. 5. Introducing energy dependence for the fluorescence rate constant we obtain good agreement with the experimental points by using an energy dependent scaling function $S(E)$, so that $k_{\mathrm{F}}(E)=S(E) 4.1 \times 10^{6} \mathrm{~s}^{-1}$, where $E$ is the excess energy in $\mathrm{S}_{1}$ :

$$
\begin{aligned}
S(E)= & \exp \left[d+a\left(E / \mathrm{cm}^{-1}-c\right)-\left(\left\{a \operatorname{abs}\left(E / \mathrm{cm}^{-1}-c\right)\right\}^{e}\right.\right. \\
& \left.\left.+b^{e}\right)^{(1 / e)}\right]+f
\end{aligned}
$$

The resulting fit in Fig. 5 (dashed line) is obtained for the parameter set $a=0.00194, b=13.60068, c=-4002.35766$, $d=2.40151, e=27.45614$ and, $f=0.98608$. The corresponding energy dependent $k_{\mathrm{F}}(E)$, which represents the correct $\Phi_{\mathrm{F}}(E)$, is plotted as a dashed line in Fig. 4.

We are now in the position to calculate the KCSF window functions via eqns. (22) and (23) and compare them with their KCSI counterparts [eqns. (16) and (17)]. ${ }^{9}$ An example is shown in Fig. 6 for $\lambda_{1}=275 \mathrm{~nm}$. Both have been normalized to a maximum amplitude of 100 . Only small differences between the KCSF window (dashed line) and the KCSI window (solid line)

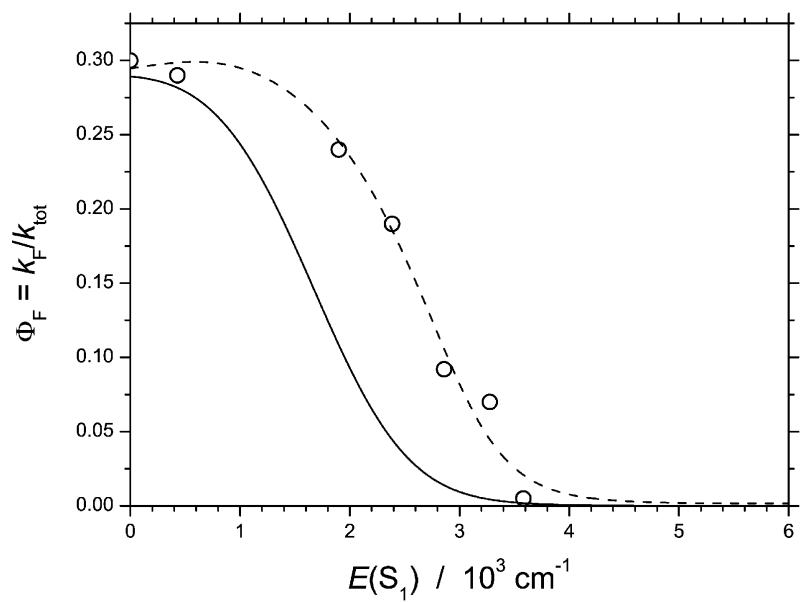

Fig. 5 Energy dependence of the fluorescence quantum yield $\Phi_{\mathrm{F}}(E)$ for toluene in the $\mathrm{S}_{1}$ state. $\bigcirc$ : Data from Burton et al., ${ }^{16,24}$ solid line: calculated on the basis of $k_{\text {tot }}(E)$ from Fig. 4 and an energy independent $k_{\mathrm{F}}=4.1 \times 10^{6} \mathrm{~s}^{-1}$, dashed line: same as the solid line, but using the energy dependent $k_{\mathrm{F}}(E)$ from Fig. 4. 


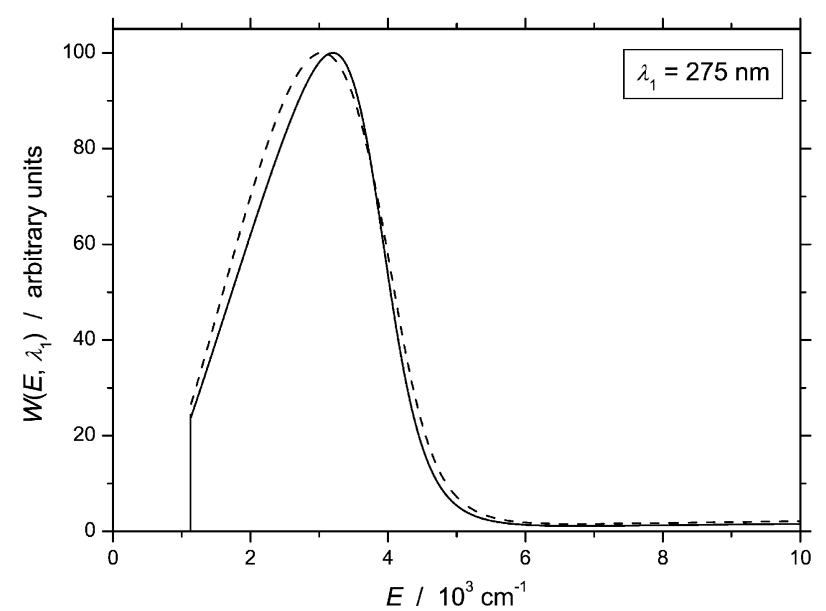

Fig. 6 Comparison of KCSF and KCSI observation windows for toluene at the probe wavelength $\lambda_{1}=275 \mathrm{~nm}$. Solid line: KCSI window function, ${ }^{9}$ dashed line: KCSF window with energy dependent $k_{\mathrm{F}}$ from Fig. 4. Note the similarity of both window shapes.

can be seen. An analogous behavior is found for the observation windows at the other probe wavelengths. Considering the fact that there are definitely certain error limits of the fluorescence quantum yields $\Phi(E)$ (which are however difficult to quantify at present) it is safe to say that the KCSF and KCSI windows for toluene have essentially the same shape. This result is consistent with the experimental findings in Fig. 2, which show that the KCSF and KCSI signals at the three probe wavelengths $\lambda_{1}$ are virtually indistinguishable. We also note at this point that the master equation simulations are rather robust against changes in the detailed shape of the window functions, as was already discussed in detail earlier. ${ }^{9}$

\section{Conclusions}

We have presented experimental results of a new technique, "kinetically controlled selective fluorescence (KCSF)", for obtaining $P\left(E^{\prime}, E\right)$ distributions characterizing CET of highly vibrationally excited molecules. The deactivation of toluene in an argon bath was studied as a test system, and referenced against data from the well-known "kinetically controlled selective ionization (KCSI)" scheme. This accurate comparison became possible using a newly designed setup, which allows simultaneous detection of fluorescence and ionization signals under identical experimental conditions ("kinetically controlled selective fluorescence and ionization = KCSFI"). The curves for toluene* + Ar have a virtually indistinguishable shape. The experimental results can be interpreted in the framework of a rate-equation approach. It was found that with typical nanosecond laser pulses $\left(t_{\mathrm{FWHM}} \approx 10 \mathrm{~ns}\right)$ and high depopulation rates in the $S_{1}$ state $\left(k_{\text {tot }}\right.$ larger than roughly $3 \times$ $10^{8} \mathrm{~s}^{-1}$ ) the yield of ions and fluorescence photons are both proportional to $k_{\mathrm{tot}}^{-1}$, the inverse of the depopulation rate constant in the intermediate state. If the absorption cross section for the second photon in the KCSI process and the fluorescence rate constant in the KCSF process are energy independent (or in the same way energy dependent) KCSF and KCSI signals will have exactly the same shape. In the toluene case however, $k_{\text {tot }}$ is fairly small at low $\mathrm{S}_{1}$ excess energies. The agreement of the KCSI and KCSF signals can then however easily be rationalized by the energy dependence of the fluorescence rate constant $k_{\mathrm{F}}$ which follows from literature fluorescence quantum yields $\Phi_{\mathrm{F}}$. Note also that collisions, which happen in the "time window" of the fluorescence detection, obviously have no influence on the KCSF signals, as underlined by our pressure dependent data.

At the current stage of the KCSF experiments it is not easy to evaluate the accuracy and uncertainty of this method compared to the KCSI technique. We believe that the results of both methods should be very similar with respect to quality and reliability. In principle, given a detailed set of KCSF curves for several observation windows, excitation wavelengths and bath gases, the results for $P\left(E^{\prime}, E\right)$ and the corresponding moments of energy transfer will become "self-calibrated". This means, that the $P\left(E^{\prime}, E\right)$ parameters and the shape of the observation windows are automatically determined from the master equation analysis. The results will therefore be independent of any external reference data, as already demonstrated in our KCSI study of azulene. ${ }^{7}$

The fluorescence detection scheme shows considerable potential for studying systems which are not easily accessible for ionization detection. For instance, fluorine substituted aromatics like hexafluorobenzene are very interesting systems because of their much higher collisional efficiency compared to their hydrocarbon analogues. ${ }^{29-31}$ However, collisional transition probabilities $P\left(E^{\prime}, E\right)$ for these systems have not yet been obtained. Unfortunately, these fluoroaromatics are characterized by high ionization potentials. ${ }^{32}$ Possible intermediate electronic states for the KCSI process are located at fairly low energies. ${ }^{33,34}$ One-color two-photon ionization is therefore not feasible, and instead a two-color two-photon scheme with two probe lasers would be required. In this case KCSF is clearly the easier approach, and we are currently exploring this avenue. In addition, preliminary results show that KCSF detection can also be successfully applied in very different experimental setups, e.g., within a Laval nozzle expansion to study the collisional deactivation of molecules at ultracold temperatures $(>50 \mathrm{~K}){ }^{35}$

\section{Acknowledgements}

We are grateful to Reinhard Bürsing for the excellent maintenance and optimization of the laser systems and his invaluable help during the setup of the new KCSFI system. In addition, we thank Vladimir Ushakov and Anatoli Maergoiz, who helped us with implementing the rate-equation model for the deduction of the KCSF window functions from their KCSI analogues. Highly purified CHT samples were prepared by Jürgen Bienert and Jens Schimpfhauser. Finally we acknowledge stimulating discussions with Jürgen Troe, Jörg Schroeder, Matthias Hollerbach, Björn Hansmann, Christian Reichardt and Kawon Oum.

\section{References}

1 H. Hippler and J. Troe, in Bimolecular Collisions, eds. J. E. Baggott and M. N. R. Ashfold, The Royal Society of Chemistry, London, 1989, p. 209

2 I. Oref and D. C. Tardy, Chem. Rev., 1990, 90, 1407.

3 M. J. Rossi and J. R. Barker, Chem. Phys. Lett., 1982, 85, 21.

4 B. M. Toselli, J. D. Brenner, M. L. Yerram, W. E. Chin, K. D. King and J. R. Barker, J. Chem. Phys., 1991, 95, 176.

5 H. Hippler, J. Troe and H. J. Wendelken, J. Chem. Phys., 1983, 78, 6709 .

6 N. Fay and K. Luther, Z. Phys. Chem., 2000, 214, 839.

7 U. Hold, T. Lenzer, K. Luther and A. C. Symonds, J. Chem. Phys., 2003, 119, 11192.

8 U. Hold, T. Lenzer, K. Luther, K. Reihs and A. C. Symonds, J. Chem. Phys., 2000, 112, 4076

9 T. Lenzer, K. Luther, K. Reihs and A. C. Symonds, J. Chem. Phys., 2000, 112, 4090.

10 R. J. Sension, S. T. Repinec and R. M. Hochstrasser, J. Chem. Phys., 1990, 93, 9185.

11 R. J. Sension, S. T. Repinec, A. Z. Szarka and R. M. Hochstrasser, J. Chem. Phys., 1993, 98, 6291.

12 L. Nikowa, D. Schwarzer and J. Troe, Chem. Phys. Lett., 1995, 233, 303 .

13 M. Hollerbach, Diploma Thesis, University of Göttingen, Göttingen, 2003.

14 H. Frerichs, M. Hollerbach and T. Lenzer and K. Luther "Indications for statistical behavior in collisional energy transfer of larger molecules", 102nd Bunsentagung, Kiel (Germany), 2003. 
15 C. Reichardt, Diploma Thesis, University of Göttingen, Göttingen, 2004.

16 C. S. Burton and W. A. Noyes Jr, J. Chem. Phys., 1968, 49, 1705.

17 W. Koban, J. D. Koch, R. K. Hanson and C. Schulz, Phys. Chem. Chem. Phys., 2004, 6, 2940.

18 W. K. Bischel, L. J. Jusinski, M. N. Spencer and D. J. Eckstrom, J. Opt. Soc. Am. B, 1985, $2,877$.

19 J. P. Reilly and K. L. Kompa, J. Chem. Phys., 1980, 73, 5468.

20 D. S. Zakheim and P. M. Johnson, Chem. Phys., 1980, 46, 263.

21 J. R. Ackerhalt and J. H. Eberly, Phys. Rev. A, 1976, 14, 1705.

22 C. L. Olson, C. A. Frost, E. L. Patterson and J. W. Poukey, Trans. Nucl. Sci., 1983, NS-30, 3189.

23 U. Grigoleit, T. Lenzer, K. Luther, M. Mützel and A. Takahara, Phys. Chem. Chem. Phys., 2001, 3, 2191.

24 M. Jacon, C. Lardeux, R. Lopez-Delgado and A. Tramer, Chem. Phys., 1977, 24, 145

25 M. Klöppel-Riech, PhD Thesis, University of Göttingen, Göttingen, 2000.
26 E. H. Kincaid, V. Worah and M. D. Schuh, J. Chem. Phys., 1991, 94, 4842.

27 C. G. Hickman, J. R. Gascooke and W. D. Lawrance, J. Chem. Phys., 1996, 104, 4887.

28 P. Farmanara, V. Stert, W. Radloff and I. V. Hertel, J. Phys. Chem. A, 2001, 105, 5613.

29 M. Damm, H. Hippler, H. A. Olschewski, J. Troe and J. Willner, Z. Phys. Chem., 1990, 166, 129.

30 T. Lenzer, K. Luther, J. Troe, R. G. Gilbert and K. F. Lim, J. Chem. Phys., 1995, 103, 626.

31 J. R. Gascooke, Z. T. Alwahabi, K. D. King and W. D. Lawrance, J. Phys. Chem. A, 1998, 102, 8505.

32 C. H. Kwon and M. S. Kim, J. Chem. Phys., 2004, 120, 11578.

33 D. V. O'Connor, M. Sumitani, J. M. Morris and K. Yoshihara, Chem. Phys. Lett., 1982, 93, 350.

34 D. Phillips, J. Chem. Phys., 1967, 46, 4679.

35 B. Abel, B. Hansmann, T. Lenzer and K. Luther, to be published. 